ARTICLE

DOI: $10.1038 / s 41467-018-05378-z$

\title{
Deep learning to predict the lab-of-origin of engineered DNA
}

\author{
Alec A.K. Nielsen ${ }^{1} \&$ Christopher A. Voigt (D) ${ }^{1}$
}

Genetic engineering projects are rapidly growing in scale and complexity, driven by new tools to design and construct DNA. There is increasing concern that widened access to these technologies could lead to attempts to construct cells for malicious intent, illegal drug production, or to steal intellectual property. Determining the origin of a DNA sequence is difficult and time-consuming. Here deep learning is applied to predict the lab-of-origin of a DNA sequence. A convolutional neural network was trained on the Addgene plasmid dataset that contained 42,364 engineered DNA sequences from 2230 labs as of February 2016. The network correctly identifies the source lab $48 \%$ of the time and $70 \%$ it appears in the top 10 predicted labs. Often, there is not a single "smoking gun" that affiliates a DNA sequence with a lab. Rather, it is a combination of design choices that are individually common but collectively reveal the designer.

\footnotetext{
${ }^{1}$ Synthetic Biology Center, Department of Biological Engineering, Massachusetts Institute of Technology, Cambridge, MA 02139, USA. Correspondence and requests for materials should be addressed to C.A.V. (email: cavoigt@gmail.com)
} 
T ed Kaczynski-the "Unabomber"-was the target of one of the longest and most expensive investigations by the FBI. He was caught when he published his 35,000-word manifesto because of similarities with earlier letters sent to his brother and newspapers: odd capitalization, linguistic idiosyncrasies, hyphen usage, and misspellings ${ }^{1}$. Individually, these features are not unique, but collectively they linked the documents. Similarly, his mail bombs shared design choices: $49 \mathrm{~V}$ batteries without outer casing, screws embedded in a wooden box, et cetera ${ }^{2}$. Again, alone these are common and widely available components, but together they pointed to a common designer.

There have been two confirmed attacks with biological weapons within the United States: the poisoning of salad bars with Salmonella in 1984 and the Anthrax letters sent in 2001. Neither involved deliberate engineering of the strain. After an insider informant pointed to the Rajneeshee cult, the subsequent attribution of the Salmonella strain relied on classical epidemiology (antibiotic susceptibility, plasmid profile, and metabolic characteristics) and a match was made to a culture tube in a clandestine $\mathrm{lab}^{3,4}$. Strains isolated from the Anthrax letters showed morphological features that were traced to genome mutations ${ }^{5}$ that aided the confirmation that the Bacillus anthracis Ames mutant was from a flask at the United States Army Medical Research Institute of Infectious Diseases at Fort Detrick ${ }^{6-8}$. Both cases involved extensive screening of large collections of natural isolates to search for strains with similar markers.

The synthetic biology era has seen rapid advances in the tools to engineer cells, leading to the forward design of large genetic systems $\mathrm{s}^{9-11}$. Building a cell to make a product, such as a material or pharmaceutical, requires the introduction of dozens of genes and genetic parts and massive changes to the genome ${ }^{12}$. There are many design choices that leave signatures in the DNA. For example, a genetic part (e.g., a promoter) might be selected from a favorite library or because of previous positive experience.
Computational design tools are increasingly prevalent ${ }^{13-23}$ and multiple options lead to subtle differences, for example, particular codon pairs that result from different gene optimization algorithms ${ }^{24-26}$. Further, DNA construction methods leave identifiable "scar" sequences ${ }^{27-30}$. Design choices can also lead to unintended markers, such as lab-specific mutants of plasmids or strains. Similarly, many commonly used genes, such as lacI or $g f p$, are reported identically in the literature but actually contain silent mutations that propagate across projects. Collectively, these design choices lead to a "signature" affiliated with an individual, lab, or institute. Even an expert eye using existing bioinformatics tools $^{31,32}$ would find it difficult or impossible to identify signatures within a long string of nucleotides (nts), such that it could be compared against large sequence databases looking for matches for attribution.

Deep convolutional neural networks (CNNs) have revolutionized image classification problems ${ }^{33}$. Layers of neurons are trained to identify signatures that parse images into categories, for example, to determine the identity of a face in a photograph from a set of possibilities ${ }^{34}$. CNNs can be trained to play checkers, $\mathrm{Go}^{35}$, and Atari games ${ }^{36}$ by processing the image of the board and selecting the next move from a set. CNNs have also been applied to categorize text based on characters, without requiring any pre-coded knowledge of words or semantic structure (e.g., news articles into "sports" and "finance") ${ }^{37}$. DNA sequences have been similarly analyzed by CNNs to identify promoters ${ }^{38}$, regulator binding sites ${ }^{37}$, and other features ${ }^{40-45}$ by training on functional and non-functional sequences. Here we apply a similar approach to train a $\mathrm{CNN}$ to categorize DNA sequences to predict the lab-of-origin ("Church," "Keasling," "Silver", et cetera) (Fig. 1a). This approach does not require any hand-selection of features or sequence-function information, such as part boundaries, operators, or gene annotation, which is often missing or inaccurate in public DNA sequence datasets. a

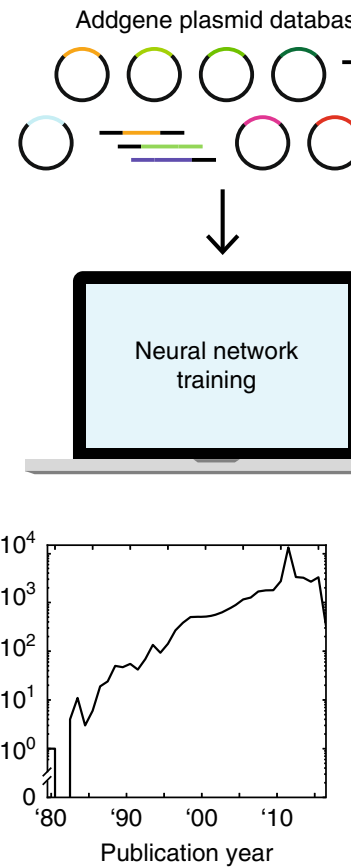

Query DNA sequence ...TGGATAAGAAATACTCAATAGG ...TGGATAAGAAATACTCAATAGG
CTTAGCTATCGGCACAAATAGCGTC GGATGGGCGGTGATCACTGATGAAT ATAAGGTTCCGTCTAAAAAGTTCAA AGAAGACAAGAAGCATGAACGTCAT CСTATTtTTGGAAATATAGTAG. . .

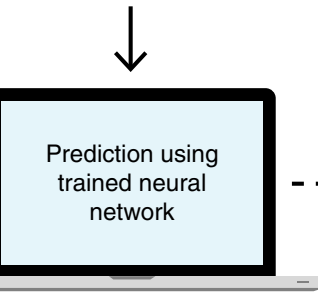

c 10

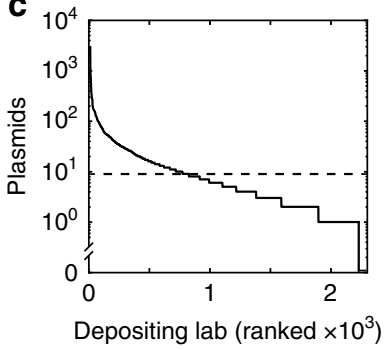

d

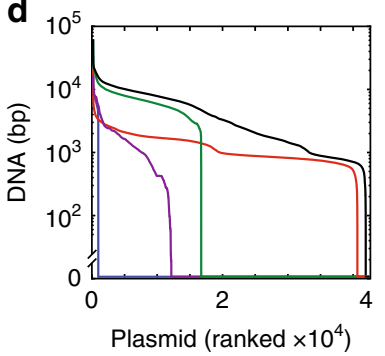

Fig. 1 Plasmid dataset and machine learning approach. a Machine learning plasmid attribution strategy. b Plasmid publication dates across the dataset. c Depositing labs ranked by how many plasmids they have submitted in the dataset. A minimum cutoff of nine plasmids was enforced for training (dashed line). d Plasmids ranked by how much associated DNA sequence (in bp) has been submitted. DNA sequence information is categorized as Partial Depositor (purple), Full Depositor (green), Partial Repository (red), and Full Repository (blue). The summed DNA sequence length across all four categories is also shown (black). Plasmid order not maintained between the five curves 


\section{Results}

Addgene dataset. The sequences of engineered DNA are maintained in a number of large public and private repositories ${ }^{46-48}$, internal databases (labs, institutes, companies, consortia), appear in published patents and papers, and in the records of DNA synthesis companies. The DNA sequences are linked with other metadata, including the submitting lab. We obtained a plasmid dataset from Addgene, a nonprofit repository that archives, maintains, and distributes plasmids to the academic community ${ }^{49}$. Globally, labs submit their plasmids for storage and redistribution (Fig. 1b-d). It contained 42,364 plasmids (197 million nts of associated DNA sequence) deposited by 2,230 labs (Methods).

Prior to analysis, we pre-processed the DNA sequence data and removed labs with fewer than nine deposited plasmids (Methods). This resulted in a set of 36,764 plasmid sequences from 827 labs. These were then divided into three groups. To ensure representation from all labs, three plasmids from each lab were selected randomly for validation and an additional three for cross-validation. The training set comprises the remaining 31,802 sequences.

Neural network architecture and performance. There are many ways to design a neural network. Initially, we tried different approaches including alternative architectures for a CNN (Supplementary Figure 1 and Supplementary Note 1A) as well as a recurrent neural network including long short-term memory units between the convolutional and fully connected layers (Supplementary Figure 2 and Supplementary Note 1B) ${ }^{43}$. A description and comparison of these methods is provided in Supplementary Information. After evaluating these approaches, we selected a $\mathrm{CNN}$ architecture (described below) that provided the best performance, is straightforward to implement, and relatively simple to interpret.

The input to the CNN is the DNA sequence encoded as a $16,048 \times 4$ matrix, where the identity of each $n t$ is represented by a one-hot vector (Fig. 2a). All sequences are 8000 nts (shorter sequences are extended with $\mathrm{Ns}$ and longer sequences are truncated) and the reverse complement is also included, separated by 48 Ns to avoid positional effects between the two. This feeds into a single convolutional layer of 128 filters, each effectively a sliding window of 12 nts. The number of convolutional layers, number of filters, and window size were determined via Bayesian optimization (Methods, Supplementary Figure 3, and Supplementary Note 1C). A max-pooling layer is applied to each filter, which reduces the size of the representation. It also eliminates the position dependence of features along the DNA sequence. The max-pooled signals from the 128 filters then feed into two fully connected layers of 64 and 827 nodes, the latter of which corresponds to the number of labs. The second fully connected layer generates outputs for each lab, which are converted to probabilities using the softmax function (Methods). These probabilities represent the relative strength of the prediction that a query DNA sequence is associated with each category (lab) and are normalized to sum to unity across categories.

This architecture consists of 1019 neurons and 69,051 parameters, which is relatively small compared to imageprocessing $\mathrm{CNNs}^{33,50,51}$. Parameterization was implemented over 100 epochs, after which a training accuracy of $77 \%$ was achieved (with a $48 \%$ validation accuracy and $48 \%$ cross-validation accuracy) (Fig. 2b). Training required $21 \mathrm{~h}$ using a single NVIDIA GRID K520 GPU. After training, the filters are conceptually similar to $12 \mathrm{nt}$ position weight matrices, a form often used in genetics for motif detection and reporting. The 128 filters are weighted differently across labs. Once trained, the $\mathrm{CNN}$ is able to rapidly analyze a sequence data stream at $980,000 \mathrm{bp} \mathrm{s}^{-1}$ on the same GPU (Methods). This is sufficiently fast for a single processor to continuously scan global DNA synthesis orders $\left(32 \mathrm{bp} \mathrm{s}^{-1}, 2015^{52}\right)$ and sequence deposits to the largest genomics database (667 bp s ${ }^{-1}$, Genbank $2016^{46}$ ).

On cross-validation sequences not used for training or validation, the $\mathrm{CNN}$ is able to predict the correct lab-of-origin $48 \%$ of the time, as ranked by activity (Fig. 2b). Random selection of a depositing lab out of the 827 would correspond to an accuracy of $0.12 \%$. Further, $70 \%$ of the time, the correct lab is ranked within the top 10 predicted labs (Fig. 2c). For a new query sequence, an activity is calculated for each lab in the set. Two examples are shown in Fig. 2 d: one has a strong prediction where the activity of the correct lab is far from the distribution and the other where the correct lab is identified, but it is much closer to the distribution. This is captured by the softmax probability, where a higher probability corresponds to a prediction that is further from the activities of other labs. Simulations are used as a means of determining the statistical significance of sequence comparisons ${ }^{53,54}$. This allows for the calculation of the likelihood that a match is random given the sequence length and size of the database. We applied this approach to calculate $P$ values associated with activities (Methods and Fig. $2 \mathrm{e}-\mathrm{h}$ ). The $P$ values for the two examples in Fig. $2 \mathrm{~d}$ are 0.0011 and 0.65 , respectively. This quantifies the significance of the first prediction, despite both having approximately the same activities.

Comparison with BLAST. The most common tool for comparing DNA sequences is BLAST (Basic Local Alignment Search Tool), designed to determine whether sequences are evolutionarily related ${ }^{31}$. BLAST seeks to identify target sequences in a database that share long stretches of identical nts with the query sequence. For our purposes, this is not ideal because many plasmids across labs share long stretches of sequences due to similar backbones, antibiotic resistances, fluorescent reporters, and so on. Searches against large databases are swamped by hits that share the long features. In contrast, the CNN focuses on short unique regions that maximally aid classification.

When BLAST is used to compare sequences with the Addgene dataset, it identifies the correct lab with $70 \%$ accuracy, slightly lower than the CNN training accuracy. The overfitting is apparent when it is used to evaluate sequences outside of training or validation, for example, from Genbank. This effect is illustrated by an example in Fig. 3 using a plasmid from the Voigt lab ( $p C I-Y F P$, JQ394803.1) that is present in Genbank but not in the Addgene dataset. This represents a hard plasmid for attribution using database information because $64 \%$ of its 3685 bp are made up of extremely common sequences (a $p 15 A$ origin of replication, $k a n R$, and $y f p$ ). There are many matches for these sequences $(E$-value $<10)$ in both the Addgene dataset/Genbank databases: $562 / 13904,502 / 3620$, and 692/1668, respectively. As such, BLAST is unable to identify the correct lab from the plasmid training dataset, from which 11,369 plasmids have an $E$-value $<10^{-180}$ and the closest Voigt lab plasmid ranking 5th (Fig. 3b). In contrast, the CNN correctly predicts $p C I-Y F P$ as being from the Voigt lab by a large difference (Fig. 3c). Further, using BLAST, we identified the next 16,000 matches from Genbank that align to $p C I-Y F P$, all of which have very low $E$-values. When these are analyzed using the $\mathrm{CNN}$, only $3 \%$ are assigned to the Voigt lab out of the 827 options (a random assignment corresponds to $0.1 \%)$.

Sensitivity to mutations. The impact of DNA sequence point mutations on the predictions was then assessed. Computationally, 
random mutations were made to the $p C I-Y F P$ plasmid and the $\mathrm{CNN}$ prediction re-evaluated. These mutations could represent sequencing errors, genetic drift, or deliberate directed evolution. On average, 316 mutations could be made to the sequence (changing $\sim 9 \%$ of the plasmid) before the correct prediction was lost (Fig. 3d). This demonstrates that the labspecific signature is robust and would be difficult to erase via a scheme of laboratory-driven neutral evolution ${ }^{55-57}$.
Interpretation of predictions. It is useful to identify the regions of a DNA sequence that correspond to its assignment to a particular lab. Note that this is not always possible, as it is notoriously difficult to interpret how neural networks make predictions $^{58}$. To this end, we developed a method to visualize regions that contribute strongly to the $\mathrm{CNN}$ prediction. Following an approach described by Solovyev and Umarov ${ }^{39}$, a window of $50 \mathrm{Ns}$ is scanned across the plasmid to obscure the underlying
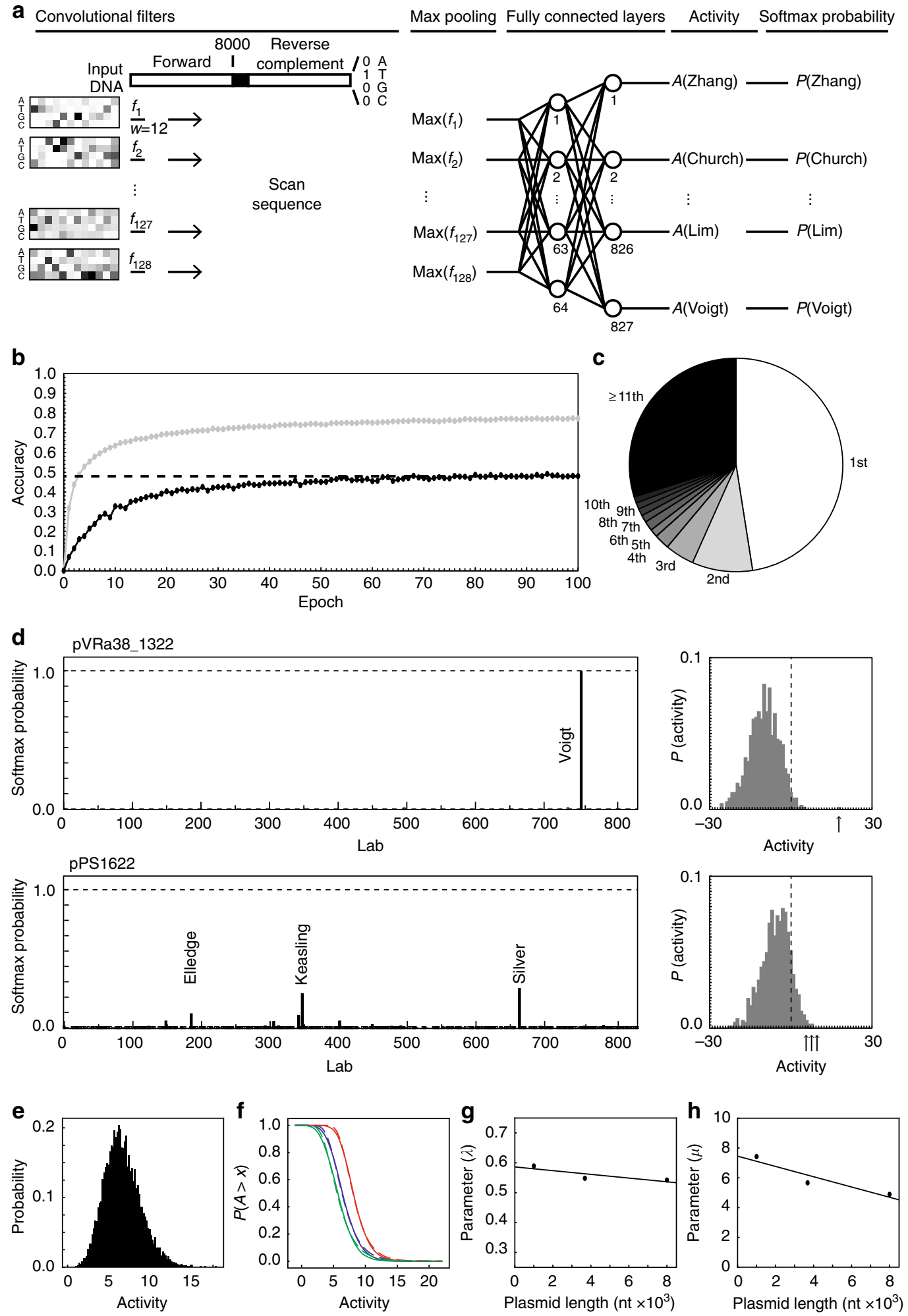
DNA sequence (Methods). The activities for the labs are calculated for each window position. A drop in the activity for a lab indicates that that sequence contributes to the prediction and if the activity goes up, it can be interpreted that this region makes it look less like it came from the lab. Note that this analysis only shows the first-order connections between sequence and the prediction and will not reveal higher-order effects requiring multiple regions (see below).

Profiles were generated for all of the plasmids tested during cross-validation. Several classes of effects were observed and representative examples are shown in Fig. 4. For most plasmids, there is no simple combination of genetic signals to guide classification, and deep learning is needed to learn complex mappings from DNA sequence. One example is the $p C I-Y F P$ plasmid, whose profile is relatively flat for the entirety of the sequence and obscuring no $50 \mathrm{nt}$ region causes it to be improperly classified (Fig. 4a).

In some cases, a "smoking gun" sequence could be identified that was strongly associated with a lab and the prediction changes drastically when this sequence is obscured. This can be from a deliberate design objective, a secondary consequence (e.g., DNA construction methodology or at part junctions), or accidentally due to a known or unknown silent mutation associated with a part (e.g., a synonymous mutation in a fluorescent reporter). An example of a deliberate design objective is the FLAG-HA-BRCC3 plasmid submitted by Wade Harper's lab (Fig. 4b). This plasmid contains a FLAG-HA affinity tag, whose presence is central to the design of the plasmid (and the "FLAG-HA" in the plasmid name). When this region of the plasmid is obscured, the plasmid can be made to look like two other labs (David Sabatini's and David Baltimore's), depending on the position of the masking window. This is due to the prevalence of other plasmid features ( $p$ BR322 origin of replication, AmpR, lac promoter, IRES, et cetera) across other labs.

An example of a consequential "smoking gun" is the $p A A V$ CAG-tdTomato (codon diversified) plasmid from Edward Boyden's lab (Fig. 4c). A region of this plasmid corresponds to a pair of restriction sites, KpnI/BamHI (3588-3599), adjacent to one of the plasmid inserts. Disrupting only $12 \mathrm{bp}$ of this sequence makes the plasmid appear as though it is from Roger Tsien's lab. Note that the origin is also associated with the Boyden lab and changing it causes the plasmid to look as though it came from the Liquin Luo lab.

Most plasmids do not have a single "smoking gun" but rather have a combination of signals that collectively contribute to the prediction. The pT7CFE1-TelR15-YPet plasmid from Thoru Pederson's lab is a good example (Fig. 4d). The lab-of-origin is correctly predicted by the particular combination of features that individually are prevalent across the Addgene dataset. If regions of the T7 promoter, yellow fluorescent protein (YPet), ampicillin resistance gene $(A m p R)$, or the $\mathrm{fl}$ origin of replication ( $f 1$ ori) are obscured, then the plasmid looks like it is from Christopher Voigt's lab. Obscuring regions of the IRES, YPet, AmpR, or f1 ori makes it look like it is from Susan Lindquist's lab. Other intergenic regions, when obscured, can make the plasmid appear to come from six different labs. Thus it is the collective impact of all these design choices that lead to a prediction, analogous to correctly identifying a noun by playing "20 questions."

\section{Discussion}

This work demonstrates that engineering DNA leaves design signatures that can be extracted from public repositories and used to guide an investigation into attribution. Over time, this will become more powerful for two reasons. First, designs in synthetic biology are growing in complexity and require more parts, genes, and genome modifications, thus leading to more "hooks" to create a unique signature ${ }^{59-61}$. Design tools are causing engineered and natural sequences to further diverge and thus easier to distinguish. Second, DNA datasets for training are growing and becoming more comprehensive, for example, Genbank doubles every 18 months and other databases are growing rapidly ${ }^{62,63}$. DNA synthesis companies, academic and industrial Bio-Foundries, and experimental cloud labs also act as centralized nodes of sequence information ${ }^{64-69,76}$.

Tracing a DNA sequence to a perpetrator requires a combination of investigative tools, some traditional and some hightech ${ }^{4}$. To this end, artificial intelligence will be a powerful tool for microbial forensics, which is currently poorly equipped to assess engineered biology. It is important to note that detecting labspecific signatures within a DNA sequence does not, in itself, indicate that that it is from that lab. Making an analogy with the Unabomber, determining a battery is from Duracell obviously does not mean that a Duracell employee is the perpetrator. Similarly, the tracing of the Samonella strain used by Rajneeshee to the commercial supplier ATCC (Rockville, MD) does not implicate ATCC but rather provides investigable clues around those that accessed or ordered it (an invoice was found at the cult compound) and points to where they may have been trained. The ability to scan engineered DNA sequences for signatures associated with individuals, labs, centers, and even countries provides actionable information to guide an investigation. Different regions of the DNA may be associated with different origins and this tool helps parse these hierarchal structures, similar to looking for shared code across computer viruses. Looking into the future, counters to this technology could also be developed, including sophisticated machine learning approaches, such as variational autoencoders $^{70}$ and generative adversarial networks ${ }^{71}$, that can

Fig. 2 Convolutional neural network accuracy. a Convolutional neural network (CNN) architecture. DNA sequences are converted to 16,048 $\times 4$ matrices, where the identity of each nucleotide is converted to a one-hot vector. This input is scanned by 128 convolutional filters $\left(f_{1}-f_{128}\right)$ each with a width, $w$, of 12 nucleotide positions. Per-position nucleotide filter weights are converted to Boltzmann factors and visualized using a heatmap (Methods). The maximum activation for each filter, $\max \left(f_{k}\right)$, across the entire input sequence is taken. Activations are fed through two fully connected layers, which generates neural activity predictions for each lab, $A(\mathrm{Name})$, before behind converted to probabilities using the softmax function, $P($ Name). The lab prediction is taken as the highest softmax probability. Batch normalization layers are not shown. $\mathbf{b}$ Training accuracy (gray) and validation accuracy (black) per epoch for the chosen architecture. Cross-validation accuracy was computed after training (dashed line). c Output prediction rank of the actual lab-of-origin for plasmids in the cross-validation set. d Neural network softmax probabilities (left panel) for a Christopher Voigt lab plasmid ( $p$ VRa38_1322) and a Pamela Silver lab plasmid (pPS1622). Labs with the highest probabilities are labeled. Normalized distribution of pre-softmax neuron activity ("Activity", right panel) for the plasmids of interest. Arrows highlight the activity for labeled labs at left. The vertical dashed lines mark the origin. e Normalized distribution of activity for $10^{4}$ random DNA sequences with length $3685 \mathrm{nt}$. f $P$ value distributions for random DNA sequences for lengths 8000,3685 , and 1000 nt (from left to right). Empirical data (solid lines) and fits to $P(A>x)=1-\exp (-\exp (-\lambda(x-\mu))$ ) (dashed lines) are shown. $\mathbf{g}$ Distribution fit steepness parameter $(\lambda)$ versus plasmid length with a trend of $\lambda=0.59-6.2 \times 10^{-6} \times$ (dashed line). $\mathbf{h}$ Distribution fit offset parameter $(\mu)$ versus plasmid length with a trend of $\mu$ $=7.5-3.4 \times 10^{-4} x$ (dashed line) 
a $\mathrm{pCI-YFP}($ accession \#: JQ394803)
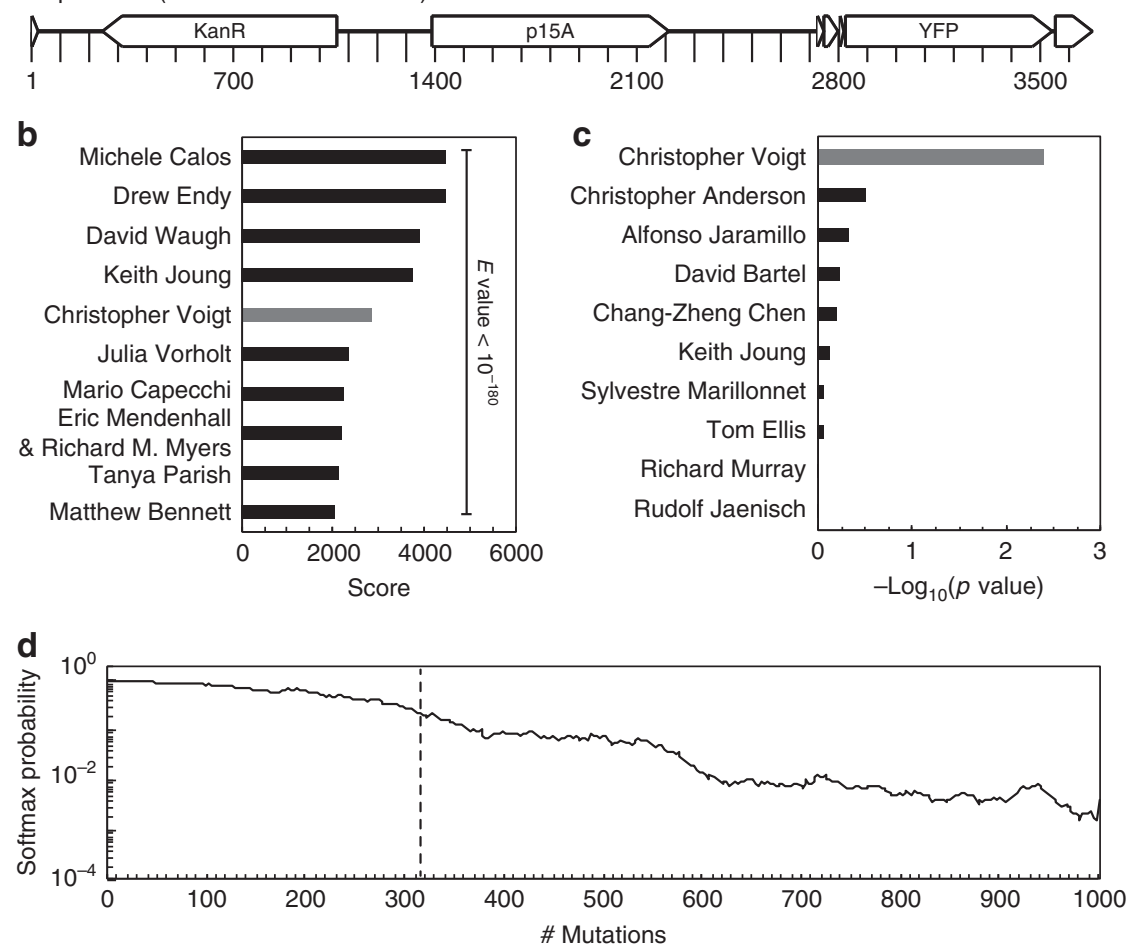

Fig. 3 Statistical analysis of neural network and BLAST results. a Plasmid map for $p C I-Y F P$ with labeled major genetic features shown. $\mathbf{b}$ BLAST score for $p C I$-YFP alignments to plasmid training set (depositing lab shown instead of plasmid name). $\mathbf{c}$ values for $p C I$-YFP lab predictions calculated from activities. d Effect of point mutations on the Voigt lab softmax probability for $\mathrm{pCl}$-YFP. The geometric mean of 30 trajectories of random mutations is shown, with the average number of mutations that cause an incorrect top prediction (dashed line)

learn how to "fool" a classifier algorithm. These approaches represent a more sophisticated version of classical approaches to avoid detection, for example, altering sequence to subvert a PCR diagnostic ${ }^{72}$. Our approach could extent to applications beyond investigating sequences of malicious intent, for example, determining that a sequence from the environment is engineered and tracing its origins. The ability to rapidly parse DNA sequence information to guide the process of attribution and the understanding of potential countermeasures are capabilities critical to a national biodefense strategy. This work is an early step in achieving this, which will ultimately require more powerful algorithms, large sequence databases, and a concerted effort to address needs for forensics and attribution ${ }^{73}$.

\section{Methods}

Plasmid dataset. Plasmid DNA sequences and metadata were obtained in the form of a JavaScript Object Notation (JSON) file obtained upon request from Addgene, dated February 2016. We parsed this file to record each plasmid's associated PubMed ID (PMID) and Digital Object Identifier (DOI). To determine a plasmid's publication year, we first attempted to locate the PMID within a locally stored manuscript ID conversion file (ftp://ftp.ncbi.nlm.nih.gov/pub/pmc/PMCids.csv.gz). If the PMID was not found, we then attempted to locate the corresponding DOI. If either the PMID or DOI was found, then the publication year was stored. If neither could be found within the locally stored file, then a PubMed lookup tool (https://pypi.python.org/pypi/pubmed-lookup) was used to request the information from the NCBI server. While slower than the local approach, we were able to locate all remaining publication dates with the exception of 15 plasmids, which had no date information provided and were excluded from Fig. 1b. We also parsed the JSON file to store each plasmid's depositing lab and associated DNA sequences. For plasmids with multiple depositing labs listed, the lab combination was treated as its own unique depositor. All unique depositors were then ranked based on their number of deposited plasmids (Fig. 1c). Plasmid DNA sequences in the JSON file came labeled as either: (1) "Full Repository", where the entire DNA sequence was submitted by Addgene, (2) "Full Depositor", where the depositing lab submitted the entire DNA sequence, (3) "Partial Repository", where one or more sections of the plasmid were submitted by Addgene, or (4) "Partial Depositor", where one or more sections of the plasmid were submitted by the depositing lab.
We summed the total number of nts associated with a plasmid in each category (and also the sum of all four categories) and then ranked the plasmids accordingly (Fig. 1d).

Input pre-processing and encoding. In order to have sufficient plasmid sequences to learn lab-of-origin from, we first eliminated any labs and their associated plasmids if the lab had deposited fewer than nine plasmids. If a plasmid had associated DNA sequence that came categorized as Full Repository, we used only that DNA sequence for training and ignored all other associated sequence information. If there was no Full Repository DNA sequence, but instead there was Full Depositor sequence, we used only that DNA sequence for training and ignored other sequences. If instead there was only Partial Repository and/or Partial Depositor DNA sequence for a plasmid (often resulting from Sanger sequencing reads), we concatenated all such partial sequences separated by spacer sequences of $48 \mathrm{Ns}$ to create the training sequence.

Subsequently, to reduce training time we truncated long DNA sequences to $8000 \mathrm{nts}$. In the rare case that any DNA sequence characters were not A, T, G, C, or $\mathrm{N}$, the character was converted to $\mathrm{N}$. We padded the resulting sequences with $\mathrm{Ns}$ to a total length of $8000 \mathrm{bp}$, and then concatenated the sequence's reverse complement to itself separated by a spacer sequence of 48 Ns. Lastly, we encoded each $n t$ in the final sequence as a one-hot vector where $A=\left[\begin{array}{llll}1 & 0 & 0 & 0\end{array}\right], T=\left[\begin{array}{llll}0 & 1 & 0 & 0\end{array}\right]$, $\mathrm{G}=\left[\begin{array}{llll}0 & 0 & 1 & 0\end{array}\right], \mathrm{C}=\left[\begin{array}{llll}0 & 0 & 0 & 1\end{array}\right]$, and $\mathrm{N}=\left[\begin{array}{llll}0 & 0 & 0 & 0\end{array}\right]$ (Fig. 2a). Similarly, the identity of the depositing lab was also encoded as a one-hot vector with length 827 . These onehot vector sequence inputs and depositor labels were used to train the neural network.

Convolutional neural network. We implemented and trained neural network architectures using Keras (version 2.0.4) using the Tensorflow backend (version 1.1.0) in Python (version 3.5.2) with NumPy (version 1.13.0) and SciPy (version 0.19.0). Other packages include Pickle for data serialization, json (version 2.0.9) to parse the Addgene data file, and pubmed_lookup (version 0.2.1). Neural networks were trained on an NVIDIA GRID K520 GPU using Amazon Web Services Elastic Cloud Compute (EC2) running Ubuntu 16.04.1 LTS.

The CNN architecture comprises from input to output: the $16,048 \times 4$ one-hot vector DNA sequence input layer, a single convolutional layer with multiple filters, a max-pooling layer for each filter over the entire input length, a batch normalization layer, a fully connected layer, a second batch normalization layer, a second fully connected layer where each node corresponds to a depositing lab, and a conversion to probability using the softmax function. Softmax is computed by 

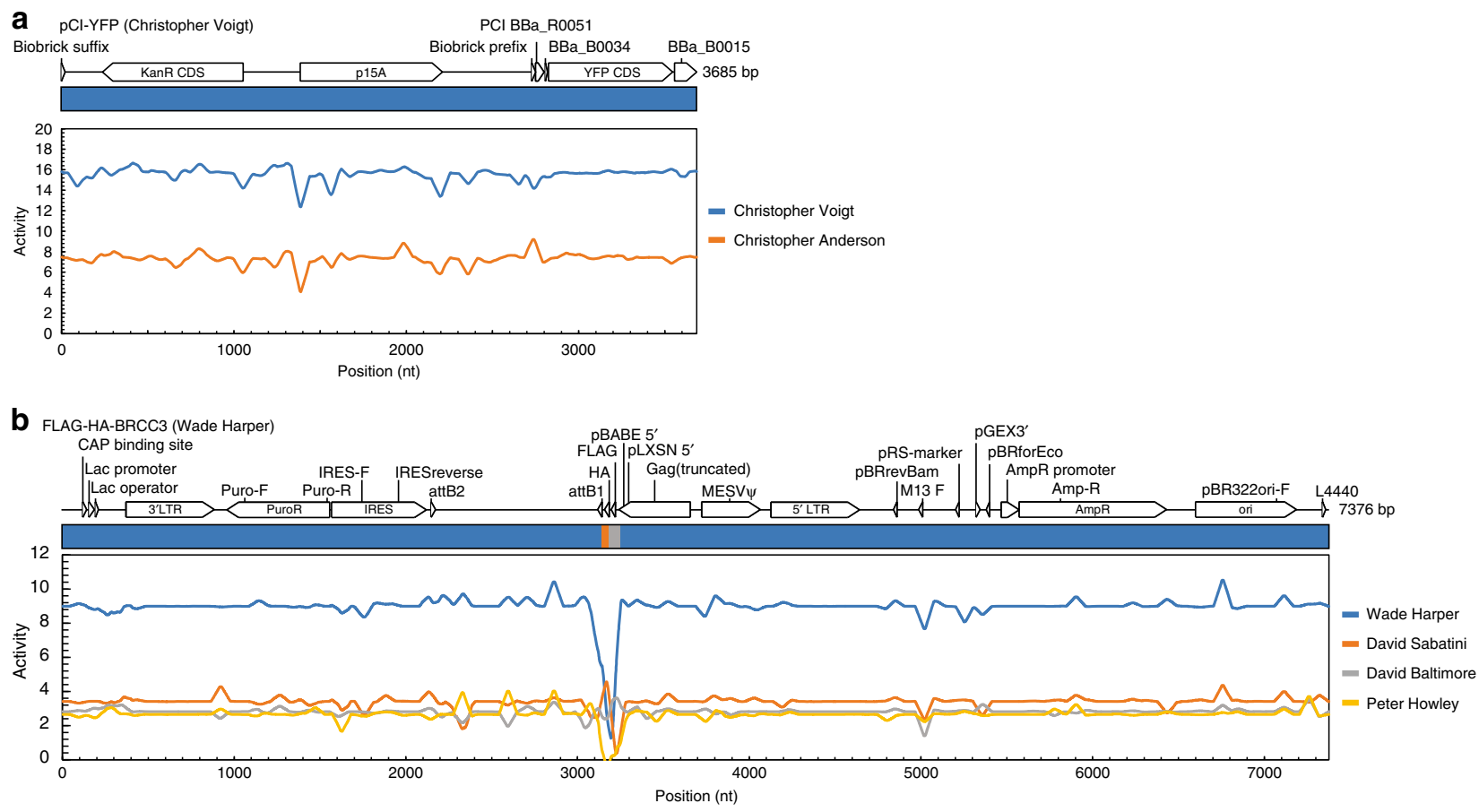

C pAAV-CAG-tdTomato (codon diversified) (Edward Boyden)
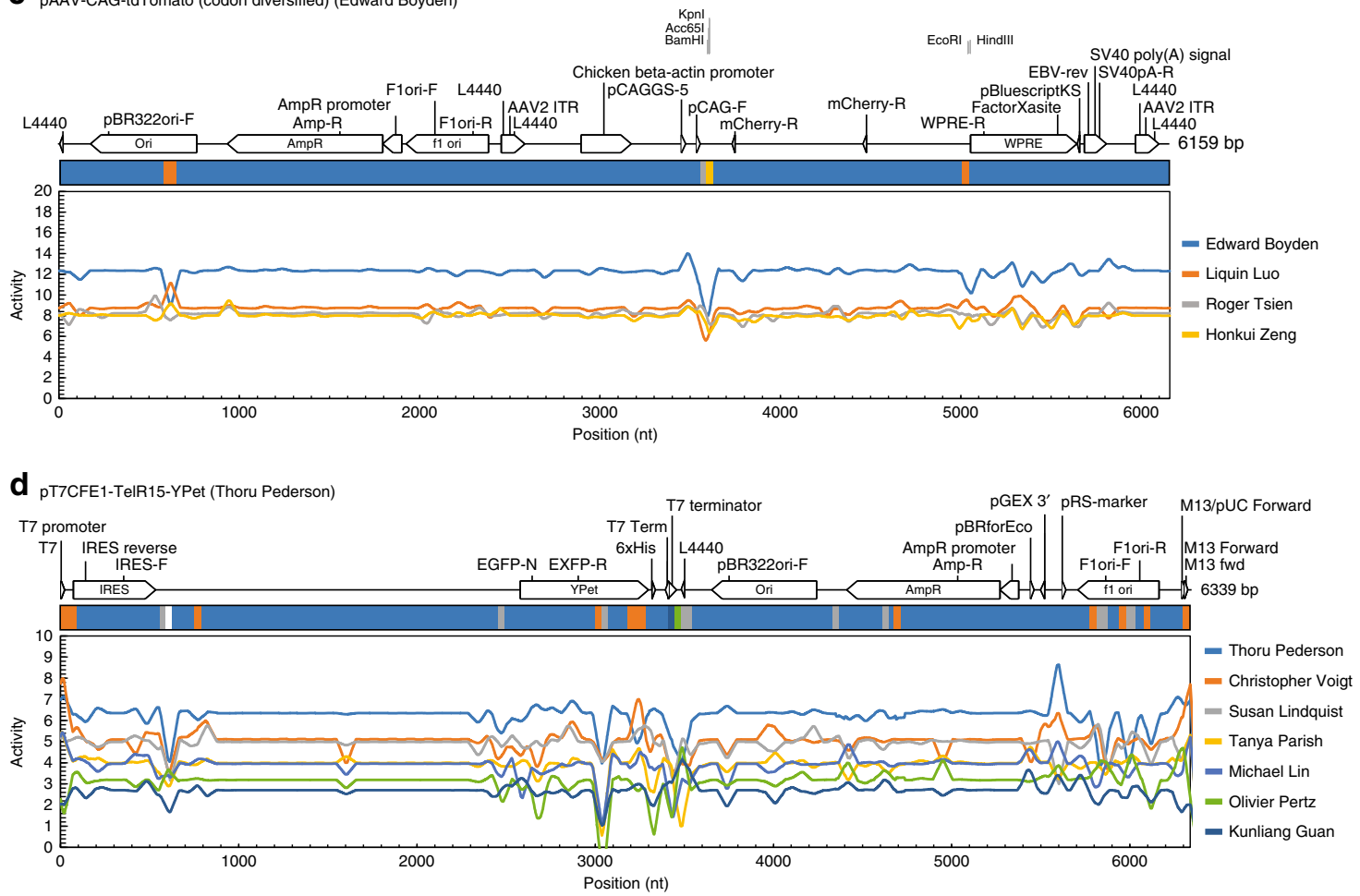

Fig. 4 Plasmid disruption analysis. Sequence scanning for plasmids a pCl-YFP from Christopher Voigt's lab, b FLAG-HA-BRCC3 from Wade Harper's lab, c pAAV-CAG-tdTomato (codon diversified) from Edward Boyden's lab, and d pT7CFE1-TelR15-YPet from Thoru Pederson's lab. Genetic schematics with feature annotations from the plasmid dataset file shown at the top of each panel. Colored bars display the lab prediction when the corresponding region of the plasmid is obscured by a sliding window of $50 \mathrm{Ns}$ (widths not to scale); colors correspond to the lab legend (right) and white corresponds to a lab not present in the legend. Colored traces show the average pre-softmax neural activity ("Activity") for top predicted labs as each region of the plasmid is obscured. For each nucleotide position, average activations are calculated for all 50 frames where the disruption window obscures that position

taking the signal $z_{j}$ generated for each lab's output node $j$ and converting to probability using the equation: $\sigma\left(z_{j}\right)=\frac{e^{e_{j}}}{\sum_{k}^{z_{k}}}$.

For the convolutional layer, we used the Keras border mode same to generate output vectors with the same size as the input. Batch normalization layers (used after the max-pool layer and the first fully connected layer) have been shown to accelerate deep network training by reducing covariate $\operatorname{shift}^{74}$. We used the rectified linear unit (ReLU) activation function for the convolutional and fully connected layers, the adam optimizer function for training, a categorical cross-entropy loss function to back-propagate errors from the output, and a minibatch size of 8 inputs per update. To compensate for skewed plasmid deposit numbers across labs, we used the Keras class_weight variable to weight 
the loss function during training by the reciprocal of training plasmid number for that lab.

Training data was split into six subsets due to memory constraints, and subsets were loaded and trained sequentially during each epoch. After training for 100 epochs, we stored the final neural network architecture and parameters for downstream use. We visualized filter weights (Fig. 2a) by converting the perposition nt filter weights $(w)$ to Boltzmann factors using the following formula, where the temperature parameter $T$ was selected by eye to maximize contrast in a heatmap: $f(w)=e^{-w / T}$

To calculate the number of DNA sequences that could be analyzed per second, we used the validation and cross-validation sets each containing 2481 plasmid sequences. Before timing, we pre-processed the input data, concatenated the sequence with $48 \mathrm{Ns}$ followed by the reverse complement sequence, and then encoded them as one-hot vectors (previous section). Using the stored neural network architecture from above, we predicted lab-of-origin for the entire encoded validation and cross-validation sets while tracking the seconds elapsed. Evaluation took $40.5 \mathrm{~s}$ to make predictions for both sets, which corresponds to 4962 sequences of $8000 \mathrm{bp}$ each, or a rate of $980,000 \mathrm{bp} \mathrm{s}^{-1}$.

Bayesian optimization of hyperparameters. To explore different hyperparameter sets for filter length, number of filters, and number of fully connected nodes, we used a Bayesian optimization technique that models the generalization performance of the hyperparameter landscape as a Gaussian process ${ }^{75}$. We used a Bayesian optimization Python package available on Github (https://github.com/ fmfn/BayesianOptimization).

We optimized for validation accuracy per wall-clock time at the end of 5 epochs using the "upper confidence bound" exploration strategy with alpha equal to $10^{-5}$ and kappa equal to 5. We varied the number of convolutional filters between 1 and 512 , the length of the convolution filters between 1 and $48 \mathrm{nts}$, and the number of nodes in the fully connected layer between 1 and 256. Hyperparameter upper bounds were chosen due to memory constraints. Training 23 different architectures yielded a range of training accuracies, validation accuracies, and wall-clock times. Many architectures failed to learn after five epochs, and these were characterized by few filters, small filter length, or both. The hyperparameters resulting in the greatest validation accuracy per training time (128 filters, a filter length of $12 \mathrm{nt}$, and 64 fully connected nodes) were selected for extended training.

Permutation of plasmid authorship. As an additional control to test for overfitting, we scrambled the depositing lab labels across all the plasmids and then split them into training, validation, and cross-validation sets. Plasmid DNA sequences were unmodified. The frequency of each lab from the non-scrambled dataset was maintained in the scrambled dataset and we used the class_weight variable in the same manner as above to compensate for differences in lab depositing frequency. We trained the network for 100 epochs, after which the validation accuracy was $0.04 \%$, comparable to what would be expected from randomly choosing a lab from the list $(0.12 \%)$.

\section{Simulation and calculation of $\boldsymbol{P}$ values. To determine the statistical}

significance of pre-softmax neuron activities generated by the CNN, we calculated $P$ values from an activity distribution for random DNA sequences ${ }^{51,52}$. We first generated $10^{4}$ random DNA sequences of length nt with the same frequencies of $\mathrm{A}, \mathrm{T}, \mathrm{G}$, and $\mathrm{C}$ as the Addgene training set. We repeated this for DNA sequence lengths $1000 \mathrm{nt}, 3685 \mathrm{nt}$ (the length of $p C I-Y F P$ ), and $8000 \mathrm{nt}$ (the maximum allowable length in our architecture). For each random DNA sequence, we padded and concatenated the sequence reverse complement in the same manner as the Addgene set (see above), before converting the sequence to a one-hot encoding. We then used the trained $\mathrm{CNN}$ to compute the maximum pre-softmax neural activity across all labs for each sequence. A normalized histogram of the max activities can be approximated by an extreme value distribution, which has a cumulative distribution function of $y=\exp (-\exp (-\lambda(x-\mu)))$. The probability of observing an activity, $A$, greater than $x$ is therefore $P(A>x)=1-\exp$ $(-\exp (-\lambda(x-\mu)))$, where $\lambda$ is the steepness parameter and $\mu$ is the distribution's offset from 0 . We fit the empirical distributions to this equation, which was then used to calculate the $P$ value of the pre-softmax neural activity for a DNA sequence of length $\mathrm{N}$.

BLAST analysis. For the alignment of the $p C I-Y F P$ sequence to sequences in Genbank, BLAST was performed against the $\mathrm{nr} / \mathrm{nt} \mathrm{nt}$ collection using the web interface (https://blast.ncbi.nlm.nih.gov/Blast.cgi). Default parameters were used except the Entrez Query is "NOT genome," Max target sequences was 20,000, Expect threshold was 0.1 , and the Max matches in a query range was 5000 . The match/mismatch scores are $1,-2$, and the gap cost was linear. For plasmid features KanR, $p 15 A$, and YFP, web BLAST was performed against the $\mathrm{nr} / \mathrm{nt}$ collection using the megablast default parameters and the number of alignments reported. Additionally, for each feature BLAST was performed locally against the training set and the number of alignments in the output file recorded. To do so, FASTA files were created for each feature, and a BLAST database was created from a FASTA file with all Addgene training DNA sequences. For a FASTA file containing the entire $p C I$-YFP sequence, BLAST was performed against the Addgene training set and the scores for the top 10 labs were recorded (Fig. 3b). The alignment of the entire $p C I-Y F P$ plasmid to the Addgene training set returned $>10^{4}$ matches. We found the smallest non-zero $E$-value $\left(4 \times 10^{-180}\right)$ and then counted the number of alignments with an $E$-value of zero to conclude that 11,369 plasmid alignments have an $E$-value $<10^{-180}$

Mutational trajectories. Point mutations were introduced to the $p C I-Y F P$ DNA sequence iteratively over 1000 generations for a single trajectory. Each point mutation in a trajectory was made at a unique position compared to all previous mutations (i.e., mutation positions were selected without replacement). The identity of the nt post-mutation was one of the three nts not present at that position pre-mutation. After each mutation, the new DNA sequence was evaluated by the fully trained neural network and the probability prediction for Christopher Voigt's lab was recorded. Thirty such mutational trajectories were performed, each starting from the original $p C I-Y F P$ sequence. The geometric mean of the probabilities at each mutation step was calculated.

Sequence scanning to determine signature. In order to determine the importance of different regions within a plasmid toward the predictions, a window of 50 Ns was scanned across the plasmid to mask the underlying sequence at each position. For each plasmid, the starting DNA sequence was the training DNA sequence before it was padded to a length of $8000 \mathrm{bp}$ and concatenated with its reverse complement. Using this sequence, a periodic boundary condition was applied so that, when any of the Ns in the $50 \mathrm{~N}$ scanning window extended past the end boundary of the DNA sequence, those Ns were placed back at the beginning of the DNA sequence. For each position of the window scan, the resulting masked DNA sequence was padded to $8000 \mathrm{bp}$, concatenated with a spacer of 48 Ns followed by the reverse complement, converted to a one-hot vector, and input into the fully trained neural network. The pre-softmax neural activities and softmax probabilities for the top predicted labs were evaluated. For each nt in a plasmid, the CNN predictions from all 50 frames where the sliding window masked that $\mathrm{nt}$ were averaged and visualized (Fig. 4, line traces). The top lab prediction for each position of the masking window was also visualized (Fig. 4, colored bars, widths not to scale).

Code availability. Source code is available from Github at https:/github.com/ VoigtLab/predict-lab-origin.

Data availability. The authors declare that all data supporting this study are available within the article and its Supplementary Information file or are available from the corresponding author upon request.

Received: 23 December 2017 Accepted: 28 June 2018 Published online: 07 August 2018

\section{References}

1. Foster, D. W. Author Unknown: On the Trail of Anonymous (Henry Holt and Co., New York City, NY, 2000).

2. Matching wits with the unabomber. Popular Science http://www.popsci.com/ technology/article/2013-04/matching-wits-unabomber\#page-3 (accessed 10 Aug 2017).

3. Török, T. J. et al. A large community outbreak of salmonellosis caused by intentional contamination of restaurant salad bars. JAMA 278, 389-395 (1997).

4. Bernett, B. C. U.S. Biodefense \& Homeland Security: Toward Detection \& Attribution (Naval Postgraduate School, Monterey, CA, 2006).

5. Read, T. D. et al. Comparative genome sequencing for discovery of novel polymorphisms in Bacillus anthracis. (Reports). Science 296, 2028-2034 (2002).

6. Rasko, D. A. et al. Bacillus anthracis comparative genome analysis in support of the Amerithrax investigation. Proc. Natl. Acad. Sci. 108, 5027-5032 (2011).

7. National Research Council. Review of the Scientific Approaches Used During the FBI's Investigation of the 2001 Anthrax Letters (The National Academies Press, Washington, DC, 2011). https://doi.org/10.17226/13098

8. Amerithrax investigative summary https://www.hsdl.org/? abstract\&did=28996 (accessed 30 Oct 2017).

9. Esvelt, K. M. \& Wang, H. H. Genome-scale engineering for systems and synthetic biology. Mol. Syst. Biol. 9, 641 (2013).

10. Church, G. M., Elowitz, M. B., Smolke, C. D., Voigt, C. A. \& Weiss, R. Realizing the potential of synthetic biology. Nat. Rev. Mol. Cell Biol. 15, 289-295 (2014).

11. National Academies of Sciences, Engineering, and Medicine. Biodefense in the Age of Synthetic Biology (The National Academies Press, Washington, DC, 2018). 
12. Meadows, A. L. et al. Rewriting yeast central carbon metabolism for industrial isoprenoid production. Nature 537, 694-697 (2016).

13. Salis, H. M., Mirsky, E. A. \& Voigt, C. A. Automated design of synthetic ribosome binding sites to control protein expression. Nat. Biotechnol. 27, 946-950 (2009).

14. Na, D., Lee, S. \& Lee, D. Mathematical modeling of translation initiation for the estimation of its efficiency to computationally design mRNA sequences with desired expression levels in prokaryotes. BMC Syst. Biol. 4, 71 (2010).

15. Beal, J. et al. An end-to-end workflow for engineering of biological networks from high-level specifications. ACS Synth. Biol. 1, 317-331 (2012).

16. Appleton, E., Tao, J., Haddock, T. \& Densmore, D. Interactive assembly algorithms for molecular cloning. Nat. Methods 11, 657-662 (2014).

17. Bhatia, S. P., Smanski, M. J., Voigt, C. A. \& Densmore, D. M. Genetic design via combinatorial constraint specification. ACS Synth. Biol. https://doi.org/ 10.1021/acssynbio.7b00154 (2017).

18. Bilitchenko, L. et al. Eugene-a domain specific language for specifying and constraining synthetic biological parts, devices, and systems. PLoS ONE 6, e18882 (2011).

19. Jang, S. S., Oishi, K. T., Egbert, R. G. \& Klavins, E. Specification and simulation of synthetic multicelled behaviors. ACS Synth. Biol. 1, 365-374 (2012).

20. Tinberg, C. E. et al. Computational design of ligand-binding proteins with high affinity and selectivity. Nature 501, 212-216 (2013).

21. Looger, L. L., Dwyer, M. A., Smith, J. J. \& Hellinga, H. W. Computational design of receptor and sensor proteins with novel functions. Nature 423, 185-190 (2003).

22. Dahiyat, B. I. \& Mayo, S. L. De novo protein design: fully automated sequence selection. Science 278, 82-87 (1997).

23. Doench, J. G. et al. Optimized sgRNA design to maximize activity and minimize off-target effects of CRISPR-Cas9. Nat. Biotechnol. 34, 184-191 (2016).

24. Gao, W., Rzewski, A., Sun, H., Robbins, P. D. \& Gambotto, A. UpGene: application of a web-based DNA codon optimization algorithm. Biotechnol. Prog. 20, 443-448 (2004)

25. Supek, F. \& Vlahoviček, K. INCA: synonymous codon usage analysis and clustering by means of self-organizing map. Bioinformatics 20, 2329-2330 (2004).

26. Jayaraj, S., Reid, R. \& Santi, D. V. GeMS: an advanced software package for designing synthetic genes. Nucleic Acids Res. 33, 3011-3016 (2005).

27. Engler, C., Kandzia, R. \& Marillonnet, S. A one pot, one step, precision cloning method with high throughput capability. PLoS ONE 3, e3647 (2008).

28. Gibson, D. G. et al. Enzymatic assembly of DNA molecules up to several hundred kilobases. Nat. Methods 6, 343-345 (2009).

29. Knight, T. Idempotent vector design for standard assembly of biobricks (MIT, Cambridge, MA, 2003)

30. Hartley, J. L., Temple, G. F. \& Brasch, M. A. DNA cloning using in vitro sitespecific recombination. Genome Res. 10, 1788-1795 (2000).

31. Altschul, S. F., Gish, W., Miller, W., Myers, E. W. \& Lipman, D. J. Basic local alignment search tool. J. Mol. Biol. 215, 403-410 (1990).

32. Gilbert, D. Bioinformatics software resources. Brief Bioinform. 5, 300-304 (2004).

33. Krizhevsky, A., Sutskever, I. \& Hinton, G. E. in Advances in Neural Information Processing Systems 25 (eds. Pereira, F., Burges, C. J. C., Bottou, L. \& Weinberger, K. Q.) 1097-1105 (Curran Associates, Inc., Red Hook, NY, 2012).

34. Parkhi, O. M., Vedaldi, A. \& Zisserman, A. Deep face recognition. BMVC 1, 6 (2015).

35. Silver, D. et al. Mastering the game of Go without human knowledge. Nature 550, 354-359 (2017)

36. Mnih, V. et al. Human-level control through deep reinforcement learning. Nature 518, 529-533 (2015).

37. Zhang, X., Zhao, J. \& LeCun, Y. in Advances in Neural Information Processing Systems 28 (eds. Cortes, C., Lawrence, N. D., Lee, D. D., Sugiyama, M. \& Garnett, R.) 649-657 (Curran Associates, Inc., Red Hook, NY, 2015).

38. Solovyev, V. \& Umarov, R. Prediction of prokaryotic and eukaryotic promoters using convolutional deep learning neural networks. arXiv:1610.00121 [Q-Bio.GN] (2016)

39. Alipanahi, B., Delong, A., Weirauch, M. T. \& Frey, B. J. Predicting the sequence specificities of DNA- and RNA-binding proteins by deep learning. Nat. Biotechnol. 33, 831-838 (2015).

40. Rosenberg, A. B., Patwardhan, R. P., Shendure, J. \& Seelig, G. Learning the sequence determinants of alternative splicing from millions of random sequences. Cell 163, 698-711 (2015).

41. Leung, M. K. K., Xiong, H. Y., Lee, L. J. \& Frey, B. J. Deep learning of the tissue-regulated splicing code. Bioinformatics 30, i121-i129 (2014).

42. Zhou, J. \& Troyanskaya, O. G. Predicting effects of noncoding variants with deep learning-based sequence model. Nat. Methods 12, 931-934 (2015).
43. Quang, D. \& Xie, X. DanQ: a hybrid convolutional and recurrent deep neura network for quantifying the function of DNA sequences. Nucleic Acids Res. 44, e107-e107 (2016)

44. Kelley, D. R., Snoek, J. \& Rinn, J. L. Basset: learning the regulatory code of the accessible genome with deep convolutional neural networks. Genome Res. $\mathbf{2 6}$, 990-999 (2016).

45. Deep learning of the regulatory grammar of yeast $5^{\prime}$ untranslated regions from 500,000 random sequences. bioRxiv https://www.biorxiv.org/content/early/ 2017/05/19/137547 (accessed 1 Oct 2017).

46. Bilofsky, H. S. \& Burks, C. The GenBank genetic sequence data bank. Nucleic Acids Res. 16(Pt A), 1861-1863 (1988).

47. Clark, K., Karsch-Mizrachi, I., Lipman, D. J., Ostell, J. \& Sayers, E. W. GenBank. Nucleic Acids Res. 44, D67-D72 (2016).

48. http://parts.igem.org. Available at: http://parts.igem.org/Main_Page (accessed 11 Jun 2017).

49. Herscovitch, M., Perkins, E., Baltus, A. \& Fan, M. Addgene provides an open forum for plasmid sharing. Nat. Biotechnol. 30, 316-317 (2012).

50. Simonyan, K. \& Zisserman, A. Very deep convolutional networks for largescale image recognition. arXiv:1409.1556 [Cs.CV] (2014).

51. Szegedy, C. et al. Going deeper with convolutions. arXiv:1409.4842 [Cs.CV] (2015).

52. Petrone, J. DNA writers attract investors. Nat. Biotechnol. 34, 363-364 (2016)

53. Altschul, S. F. \& Gish, W. Local alignment statistics. Methods Enzymol. 266, 460-480 (1996)

54. Waterman, M. S. \& Vingron, M. Rapid and accurate estimates of statistical significance for sequence data base searches. Proc. Natl. Acad. Sci. USA 91, 4625-4628 (1994).

55. Bloom, J. D. \& Arnold, F. H. In the light of directed evolution: pathways of adaptive protein evolution. Proc. Natl. Acad. Sci. 106, 9995-10000 (2009).

56. Avoigt, C., Kauffman, S. \& Wang, Z.-G. Rational evolutionary design: the theory of in vitro protein evolution. Adv. Protein Chem. 55, 79-160 (2001).

57. Eigen, M., McCaskill, J. \& Schuster, P. in Advances in Chemical Physics (eds. Prigogine, I. \& Rice, S. A.) 149-263 (John Wiley \& Sons, Inc., New York, Chichester, 1989). https://doi.org/10.1002/9780470141243.ch4

58. Montavon, G., Samek, W. \& Müller, K.-R. Methods for interpreting and understanding deep neural networks. Digit. Signal Process. 73, 1-15 (2018).

59. Boeke, J. D. et al. The Genome Project-Write. Science 353, 126-127 (2016).

60. Annaluru, N. et al. Total synthesis of a functional designer eukaryotic chromosome. Science 344, 55-58 (2014).

61. Hutchison, C. A. et al. Design and synthesis of a minimal bacterial genome. Science 351, aad6253 (2016).

62. https://synberc-registry.jbei.org/. Available at: https://synberc-registry.jbei.org/ login (accessed 11 Jun 2017).

63. https://registry.jgi-psf.org/. Available at: https://registry.jgi-psf.org/login (accessed 11 Jun 2017).

64. The Organism Company. Ginkgo Bioworks. Available at: http://www. ginkgobioworks.com/ (accessed 29 Oct 2017).

65. Zymergen. Available at: https://www.zymergen.com/ (accessed 15 Jul 2018)

66. Twist Bioscience / We lead innovation in DNA synthesis. Available at: https:// twistbioscience.com/ (accessed 29 Oct 2017)

67. GenScript-Make Research Easy-The leader in molecular cloning and gene synthesis, peptide synthesis, protein and antibody engineering. Available at: https://www.genscript.com/ (accessed 29 Oct 2017).

68. Integrated DNA Technologies-Home. Available at: https://www.idtdna.com/ site (accessed 29 Oct 2017)

69. Casini, A. et al. A pressure test to make 10 molecules in 90 days: external evaluation of methods to engineer biology. J. Am. Chem. Soc. 140, 4302-4316 (2018).

70. Kingma, D. P. \& Welling, M. Auto-encoding variational Bayes. arXiv:1312.6114 [Cs.Stat] (2013)

71. Goodfellow, I. et al. in Advances in Neural Information Processing Systems 27 (eds. Ghahramani, Z., Welling, M., Cortes, C., Lawrence, N. D. \& Weinberger, K. Q.) 2672-2680 (Curran Associates, Inc., Red Hook, NY, 2014).

72. Higgins, J. A. et al. Sensitive and Rapid Identification of Biological Threat Agents (Army Medical Research Institute of Infectious Diseases, Fort Detrick, MD, 1999).

73. Budowle, B. et al. Toward a system of microbial forensics: from sample collection to interpretation of evidence. Appl. Environ. Microbiol. 71 2209-2213 (2005).

74. Ioffe, S. \& Szegedy, C. Batch normalization: accelerating deep network training by reducing internal covariate shift. arXiv:1502.03167 [Cs.LG] (2015).

75. Snoek, J., Larochelle, H. \& Adams, R. P. in Advances in Neural Information Processing Systems 25 (eds. Pereira, F., Burges, C. J. C., Bottou, L. \& Weinberger, K. Q.) 2951-2959 (Curran Associates, Inc., Red Hook, NY, 2012).

76. Asimov - Bring Computation to Life. Asimov Inc. Available at: http://www. asimov.io/ (accessed 15 Jul 2018). 


\section{Acknowledgements}

C.A.V. is supported by US Defense Advanced Research Projects Agency (DARPA) Living Foundries awards HR0011-13-1-0001 and HR0011-15-C-0084. A.A.K.N received Government support under FA9550-11-C-0028 and awarded by the Department of Defense, Air Force Office of Scientific Research, National Defense Science and Engineering Graduate (NDSEG) Fellowship, 32 CFR 168a, and a scholarship from the Siebel Scholars Foundation.

\section{Author contributions}

Both the authors conceived the study and wrote the manuscript. A.A.K.N. performed the computational studies and analyzed the data. C.A.V. managed the project.

\section{Additional information}

Supplementary Information accompanies this paper at https://doi.org/10.1038/s41467018-05378-z.

Competing interests: C.A.V. and A.A.K.N. are shareholders in Asimov, Inc.

Reprints and permission information is available online at http://npg.nature.com/ reprintsandpermissions/
Publisher's note: Springer Nature remains neutral with regard to jurisdictional claims in published maps and institutional affiliations.

\section{(c) (1)}

Open Access This article is licensed under a Creative Commons Attribution 4.0 International License, which permits use, sharing, adaptation, distribution and reproduction in any medium or format, as long as you give appropriate credit to the original author(s) and the source, provide a link to the Creative Commons license, and indicate if changes were made. The images or other third party material in this article are included in the article's Creative Commons license, unless indicated otherwise in a credit line to the material. If material is not included in the article's Creative Commons license and your intended use is not permitted by statutory regulation or exceeds the permitted use, you will need to obtain permission directly from the copyright holder. To view a copy of this license, visit http://creativecommons.org/ licenses/by/4.0/.

(C) The Author(s) 2018 